\title{
Design and modelling of an energy harvester for tire pressure monitoring systems
}

\author{
E. Zaouali ${ }^{1}$, F. Najar ${ }^{1}$, N. Kacem², and E. Foltete ${ }^{2}$ \\ ${ }^{1}$ LASMAP, Tunisia Polytechnic School, University of Carthage, La Marsa, Tunisia \\ ${ }^{2}$ FEMTO-ST, Université de Franche-Comté, Besançon, France
}

\begin{abstract}
The main objective of this work is to design a kinetic energy harvester for TPMS applications. The energy harvester presented in this work is a multi-pendulum fixed to a rolling wheel, which exploits nonlinear effects rendered by such a design.
\end{abstract}

\section{Introduction}

In many cases, it is not possible to supply a sensor network by means of batteries because their life is too short compared to the life of the sensor, and their replacement may be not advisable, dangerous, too expensive or impossible. In these situations, one possible solution is the use of an energy harvester, a device designed to collect energy from a source available in the environment. Indeed, it is possible to collect energy from multiple sources such as radiation, temperature gradient, movement and vibrations.

One of the promising applications of sensor networks are TPMS (or Tire Pressure Monitoring System) where energy harvester are used to extract energy from rotating wheels.

In the literature, the fact that these systems are installed in the wheels, led researchers to use different sources such as tire deformation, variation of the radial acceleration due to wheel contact with the ground, vibration, pressure variation, rotation.

From the literature, we note that the key factor in the choice of designs is the way in which the collectors are fixed: on the tire or the rim.

For the first type Lee et al. [1] studied a patch of piezofibers with interdigitated electrodes and used the tire deformation during the rotation to harvest energy. Singh et al. [2] designed a piezoelectric cantilever beam with a mass placed on the free and Tornincasa et al. [3] used the high amplitude accelerations during the rotation of the tire and a permanent magnet sliding into coils as a transducer.

For the second type Gu et al. [4] designed an energy harvester made of two beams; a piezoelectric generating beam and a flexible driving beam with a mass mounted at the free end.
Wang et al. [5] proposed an energy harvester using a rotating Halbach array magnetic disk to convert the kinetic energy of the rim to electrical power.

In this work, we propose to exploit the kinetic energy produced by a rotating wheel and convert it through electromagnetic transduction to electrical energy. We choose, as a design, a multi-pendulum system fixed to the rim. The transducer mechanism is mounted directly at the revolute joints.

\section{Different pendulum designs}

\subsection{Double Pendulum with non-parallel axes}

Consider a disk of centre $\mathrm{O}$, radius $R$, mass $m_{1}$, rotating about an axis $z_{0}$ of an inertial frame $\left(O_{0}, x_{0}, y_{0}, z_{0}\right)$ and a local coordinate $\left(O, x_{0}, y_{0}, z_{0}\right)$ in translation relatively to the Galilean reference. A rod is attached to the perimeter of the disk according to its radial direction along an axis $x_{1}$. Consider a pendulum, consisting of a rigid axis and a mass $m_{2}$, mounted about a revolute joint around an axis, itself mounted to the rod about a rigid joint, with an angle $\alpha$ (see Figure 1).

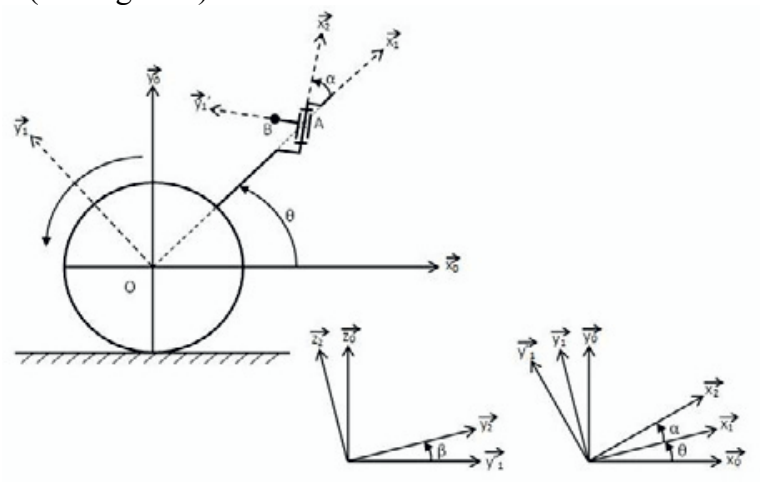


Fig. 1. Double pendulum system with non-parallel axes. Consider $m_{1}>>m_{2}$ and $B$ the center of mass.

Define a local coordinate $\left(O, x_{1}, y_{1}, z_{0}\right)$, attached to the disk, and a local coordinate $\left(A, x_{2}, y_{2}, z_{2}\right)$, attached to the pendulum. That gives:

$$
\overrightarrow{O_{0} B}(t)=v_{0} t \vec{x}_{0}+a \vec{x}_{1}+r \vec{y}_{2}
$$

where $v_{0}$ is the linear velocity of the wheel.

Consider the Lagrangian $L=T-V$, with $T$ the kinetic energy and $V$ the potential energy. The Lagrange equations are given by:

$$
\frac{d}{d t} \frac{\partial L}{\partial \dot{\beta}}-\frac{\partial L}{\partial \beta}=0
$$

Adding a damping term to the above equation leads to the following equation of motion:

$$
\begin{aligned}
& \ddot{\beta}(t)+\mathrm{c} \dot{\beta}(t)+\frac{1}{2} \dot{\theta}^{2} \sin (2 \beta(t))+\mathrm{m}_{2}\left[(b \cos (\alpha)-a \sin (\alpha)) \dot{\theta}^{2}\right. \\
& +g(\sin (\alpha) \sin (t \dot{\theta})-\cos (\alpha) \cos (t \dot{\theta}))] \sin (\beta(t))=0
\end{aligned}
$$

The damping coefficient $c$ will model both the structural damping and the electromagnetic damping due to the electrical impedance of the harvesting circuit.

\subsection{Triple Pendulum with parallel axes}

In this case of study, we consider a double pendulum, consisting of a rigid axes and a mass $\mathrm{m} 2$, mounted to the wheel, such that all axes of rotation are parallel (see Figure 2).

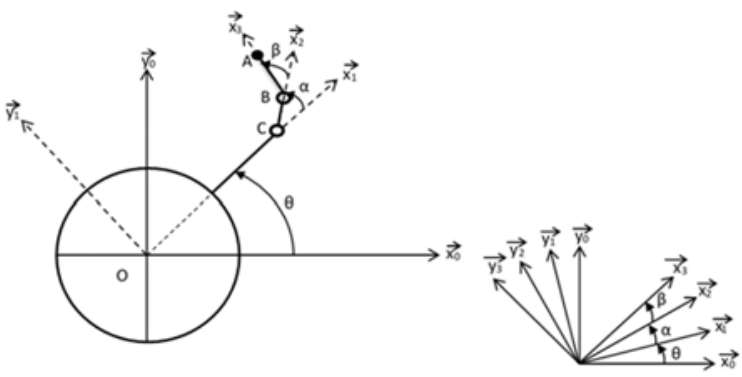

Fig. 2. Triple pendulum system with parallel axes. Consider $m_{1}>>m_{2}$ and $A$ the centre of mass.

Define a local coordinate $\left(O, x_{1}, y_{1}, z_{0}\right)$, attached to the disk, a first local coordinate $\left(C, x_{2}, y_{2}, z_{0}\right)$, attached to the first pendulum, and a second local coordinate $\left(B, x_{3}, y_{3}, z_{0}\right)$ attached to the second pendulum. That gives:

$$
\overrightarrow{O_{0} A}=v_{0} t \vec{x}_{0}+R \vec{x}_{1}+r\left(\vec{x}_{2}+\vec{x}_{3}\right)
$$

Note that, in this case, the equation of motion is too complicated to be presented here.

\section{Numerical simulation}

In each case of study, consider that: the disk reaches its rotation speed $\theta^{\prime}$ after a period of 10 seconds (Fig. 3), the rim diameter is 15 inches $(38.1 \mathrm{~cm})$, the tire is type 195/65/R15 (a thickness equals to $126.75 \mathrm{~mm}$ ), gives a total radius of $317.25 \mathrm{~mm}$ and a circumference of $1.99 \mathrm{~m}$.

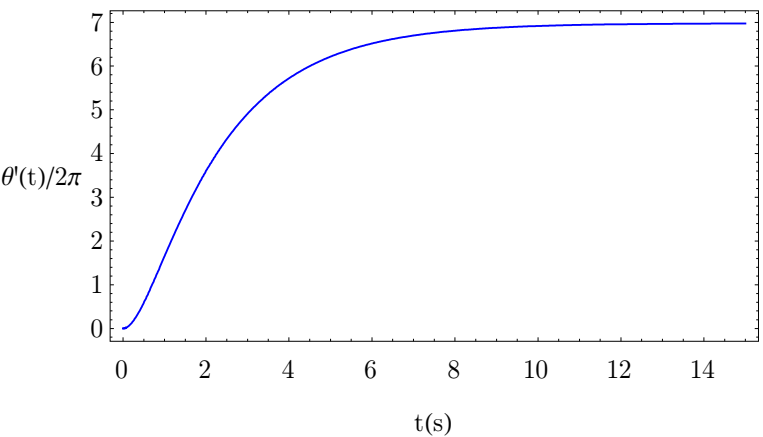

Fig. 3. Variation of the wheel rotation speed from 0 to $50 \mathrm{~km} / \mathrm{h}$.

To convert the kinetic energy into electrical energy, we use an electromagnetic transduction. We need to calculate the maximum of angular velocity of the pendulum, i.e. the variation of $\alpha^{\prime} \& \beta^{\prime}$ in function of $\theta$, to determine the power generation. The dynamic behavior of the pendulum exhibits nonlinear phenomena and can be acquired by solving equations of motion using RungeKutta method in Mathematica.

\subsection{Double Pendulum with non-parallel axes}

Figure 3 shows the variation of angular velocity under $c=0$ and wheel rotation maximum speed equals to $50 \mathrm{~km} / \mathrm{h}$ ( $\dot{\theta}=3.49$ rps i.e. $21.94 \mathrm{rd} / \mathrm{s}$ ).

Figure 4 shows the variation of angular velocity under different values of damping coefficient and wheel rotation maximum speed equals to $50 \mathrm{~km} / \mathrm{h}$.

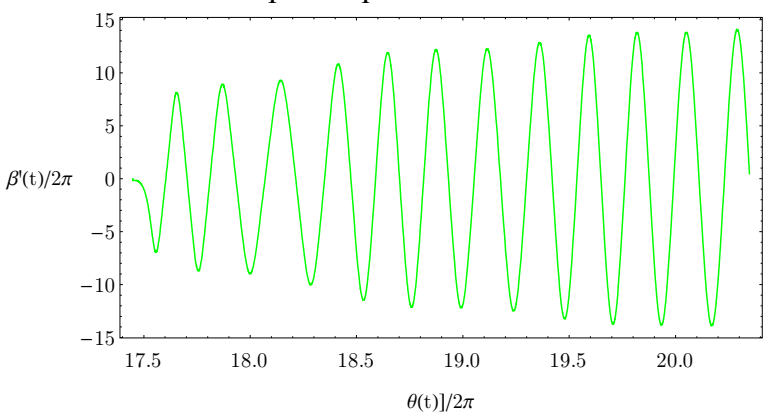

Fig. 4. Numerical simulation of the angular velocity for $c=0$.

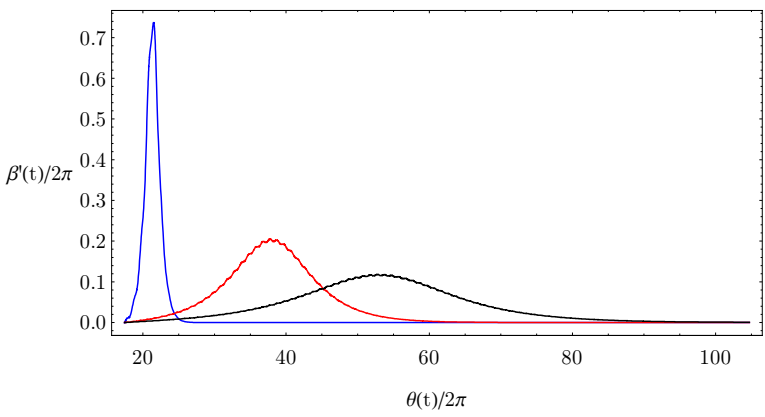

Fig. 5. Numerical simulation of the angular velocity for different values of $c$ (blue curve for $c=0.1 \mathrm{~N} \mathrm{~s} / \mathrm{kg} / \mathrm{m}$, red curve for $c=1 \mathrm{~N} \mathrm{~s} / \mathrm{kg} / \mathrm{m} \&$ black curve for $c=2 \mathrm{~N} \mathrm{~s} / \mathrm{kg} / \mathrm{m}$ ). 


\subsection{Triple Pendulum with parallel axes}

For this type of pendulum, we have different probabilities, depending on how we place the transduction mechanism. Consider $c_{1}$ is the damping coefficient related to $\mathrm{C}$ (angular velocity $\alpha^{\prime}$ ) and $c_{2}$ the damping coefficient related to $\mathrm{B}$ (angular velocity $\beta^{\prime}$ ). That gives three cases under study:

Case 1: $c_{1}=0$ and $c_{2}=1$ (Figure 6).

Case2: $c_{l}=1$ and $c_{2}=0$ (Figure 7).

Case $3: c_{1}=1$ and $c_{2}=1$ (Figure 8).

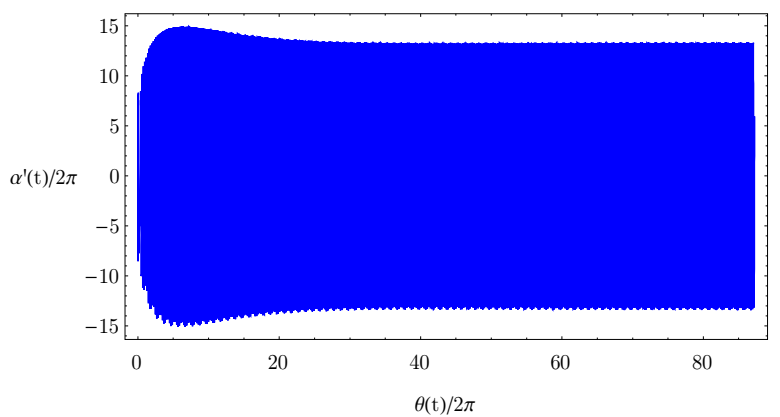

(a)

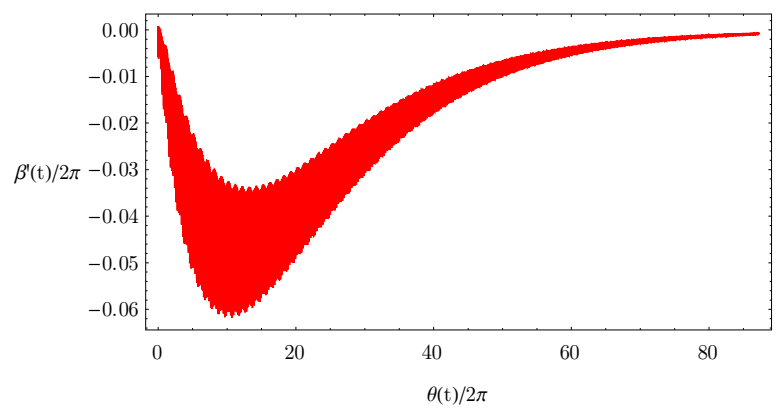

(b)

Fig. 6. Numerical simulation of the angular velocities for $\mathrm{c} 1=0$ and $\mathrm{c} 2=1$. (a) angular velocity in $\mathrm{C}$, (b) angular velocity in $\mathrm{B}$.

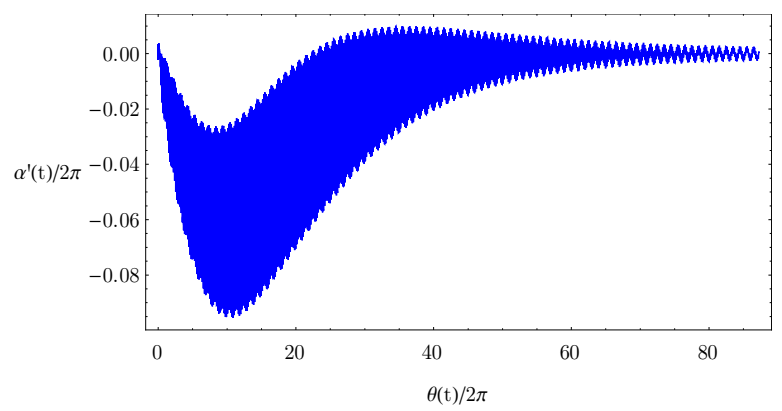

(a)

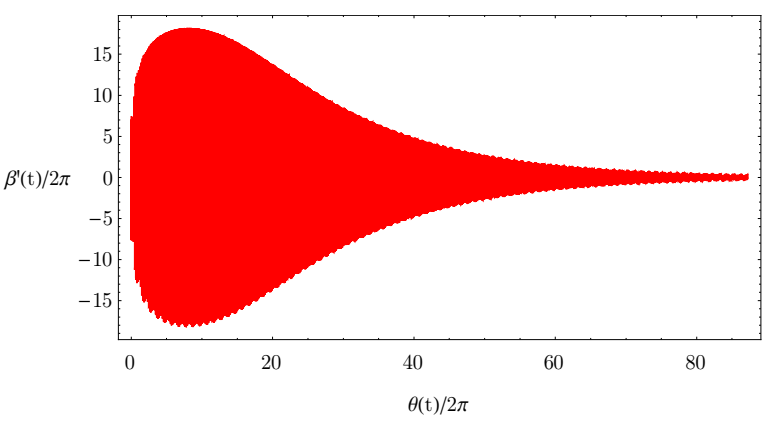

(b)

Fig. 7. Numerical simulation of the angular velocities for $\mathrm{c} 1=1$ and $\mathrm{c} 2=0$. (a) angular velocity in $\mathrm{C}$, (b) angular velocity in $\mathrm{B}$.

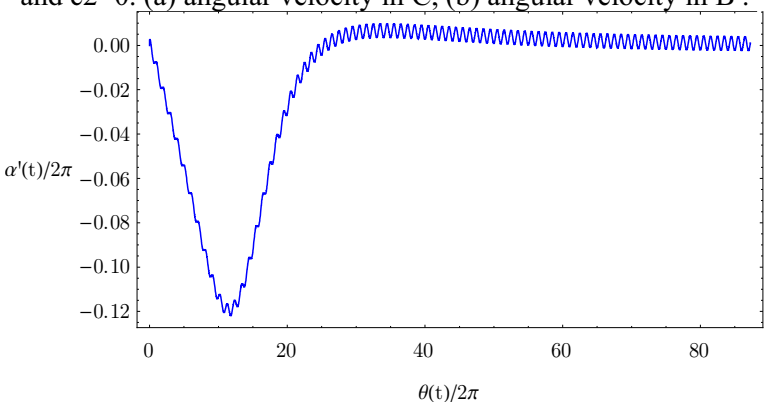

(a)

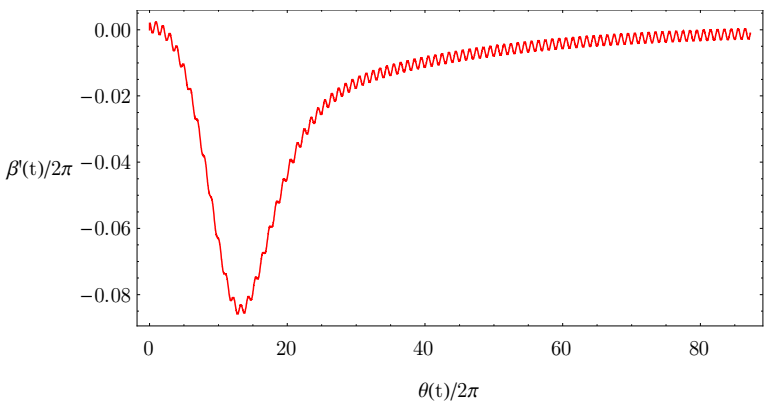

(b)

Fig. 8. Numerical simulation of the angular velocities for $\mathrm{c} 1=1$ and $\mathrm{c} 2=1$. (a) angular velocity in $\mathrm{C},(\mathrm{b})$ angular velocity in $\mathrm{B}$. To compare the performances of different designs, we can estimate the power generated $P$ in each case by taking

$$
P=R_{e s} C_{e m} \int \dot{\theta}^{2} d t
$$

with $R_{e s}$ the resistance of the electromagnetic transducer and $C_{e m}$ the electromagnetic damping coefficient.

Noting that $P$ is proportional to the RMS value of the velocity, we calculate this later for each design, taking into consideration the sensitivity to the initial condition. For this we start by choosing different wheel angular positions $\theta$ and calculate the corresponding pendulum's positions, as initial condition. Then we calculate the RMS value of the velocity for each starting position when $c=1$ (Figure 8).

Finally, we take the maximum RMS value of each design (Table 1 \& Table2). 


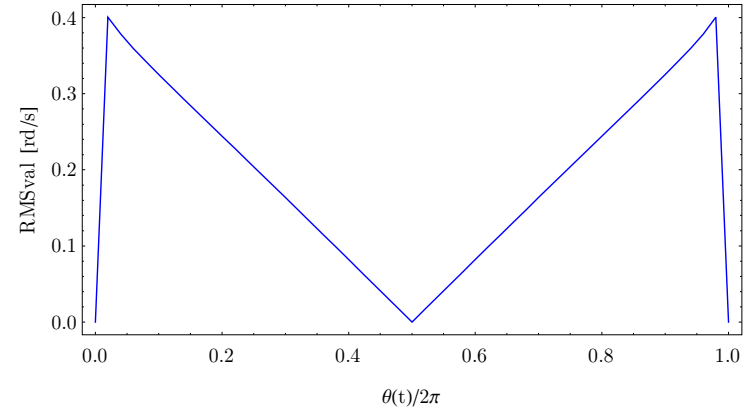

(a)

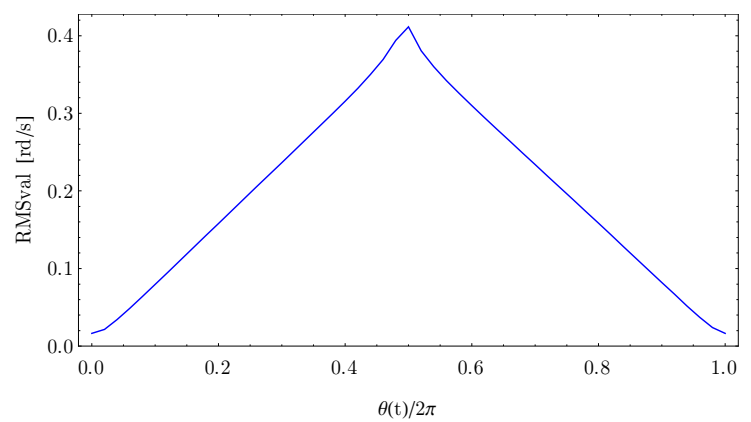

(b)

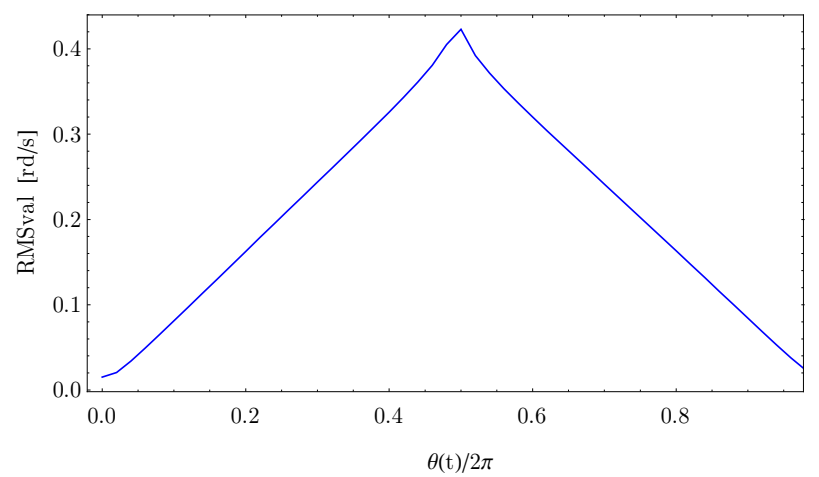

(c)

Fig. 8. Numerical simulation of the velocity RMS value for different wheel angular positions: (a) double pendulum with non-coplanar axes, (b) Triple pendulum with parallel axes, (c) double pendulum with parallel axes.

\begin{tabular}{|l|c|c|c|}
\cline { 2 - 4 } \multicolumn{1}{c|}{} & $\begin{array}{c}\text { Case 1 } \\
\text { (according } \\
\left.\text { to } \beta^{\prime}\right)\end{array}$ & $\begin{array}{c}\text { Case 2 } \\
(\text { according } \\
\left.\text { to } \alpha^{\prime}\right)\end{array}$ & $\begin{array}{c}\text { Case 3 } \\
(\text { according } \\
\left.\text { to } \alpha^{\prime}+\beta^{\prime}\right)\end{array}$ \\
\hline $\begin{array}{l}\text { Max } R M S_{\text {Value }}=T\left(\frac{P}{R_{e s} C_{e m}}\right)^{2} \\
{[\mathrm{rd} / \mathrm{s}]}\end{array}$ & $0.1610^{-8}$ & 0.33 & 0.41 \\
\hline
\end{tabular}

Table 1. Maximum RMS values for different designs of a triple pendulum with parallel axes.

\begin{tabular}{|l|c|c|c|}
\cline { 2 - 4 } \multicolumn{1}{c|}{} & DPNPA & TPPA & DPPA \\
\hline $\begin{array}{l}\text { Max } R M S_{\text {Value }}=T\left(\frac{P}{R_{e s} C_{e m}}\right)^{2} \\
{[\mathrm{rd} / \mathrm{s}]}\end{array}$ & 0.4 & 0.41 & 0.42 \\
\hline
\end{tabular}

Table 2. Velocity RMS values for different pendulums.

\section{References}

[1] J. Lee, S. Kim, J. Oh, B. Choi, A self-powering system based on tire deformation during driving, International Journal of Automotive Technology, Vol. 13, $\mathrm{N}^{\circ} 6$, pp.963-969 (2012).

[2] K B Singh, V Bedekar, S Taheri, S Priya, Piezoelectric vibration energy harvesting system with an adaptive frequency tuning mechanism for intelligent tires Mechatronics, Vol22, 2012, Pp 970-988.

[3] S Tornincasa, M Repetto, E Bonisoli, F Di Monaco, Energy harvester for vehicle tires: Nonlinear dynamics and experimental outcomes, Journal of Intelligent Material Systems and Structures, vol. 23, pp 3-13, (2012).

[4] L Gu and C Livermore, Compact passively selftuning energy harvesting for rotating applications, Smart materials and structures 21015002 (9pp), 2012.

[5] Y-J Wang, C-D Chen, C-K Sung, C Li, Natural frequency self-tuning energy harvester using a circular Halbach array magnetic disk, Journal of Intelligent Material Systems and structures, 23(8) 933-943, 2012. 